Vol. 17 (2008): 177-190.

\title{
The effects of selenate and sulphate supply on the accumulation and volatilization of Se by cabbage, kohlrabi and alfalfa plants grown hydroponically
}

\author{
Roghieh Hajiboland \\ Plant Science Department, University of Tabriz, 51666 Tabriz, Iran, \\ Excellent Center of Biodiversity, University of Tabriz, 51666 Tabriz, Iran, \\ e-mail:ehsan@tabrizu.ac.ir \\ Leila Amjad \\ Plant Science Department, University of Tabriz, 51666 Tabriz, Iran
}

\begin{abstract}
The effect of Selenium (Se) supplementation at five levels of 0 (control), 5, 10, 15, $20 \mu \mathrm{M}$ in plants supplied with one of four concentrations of sulphur (S) including $0.05,0.25,0.5$ and $1.0 \mathrm{mM}$ was investigated in two varieties of Brassica oleracea (cabbage and kohlrabi) and alfalfa (Medicago sativa $\mathrm{L}$.) in a hydroponic experiment. In severely $\mathrm{S}$ deficient plants $(0.05 \mathrm{mM})$, Se acted as a toxic element, alfalfa was the most susceptible plant that died at this treatment. However, in plants supplied with near adequate $(0.5 \mathrm{mM})$ or adequate $(1.0 \mathrm{mM}) \mathrm{S}$, Se acted as a growth promoting element. The most pronounced stimulation of growth was observed in cabbage and the lowest in alfalfa. Increasing S concentration in the medium, reduced Se uptake and transport. In contrast, $\mathrm{S}$ uptake and transport increased in response to Se addition. Se volatilization was higher in alfalfa than cabbage and kohlrabi when expressed on unit shoot dry weight or leaf area basis, but not when expressed per plant. Results suggested that Se supplementation of plants supplied with adequate S, not only had beneficial effects on plants growth but also can have application in enrichment of livestock fodder and human food.
\end{abstract}

Key-words: Alfalfa, Brassica oleraceae, Cabbage, Kohlrabi, Medicago sativa, Selenium supplementation, Sulphur, Transport, Uptake. 
Hajiboland, R. \& Amjad, L. Accumulation and volatilization of selenium in two Brassica varieties

\section{Introduction}

Selenium (Se) is one of the most widely distributed elements in the earth. A considerable variability exists for its soil concentration from location to location (Mayland et al. 1989). Se has valencies of-II (selenide, $\mathrm{Se}^{2-}$ ), 0, IV (selenite, $\mathrm{SeO}_{3}{ }^{2-}$ ) and $\mathrm{VI}$ (selenate, $\mathrm{SeO}_{4}^{2-}$ ). From both soils and nutrient solutions plants take up selenate in strong preference to selenite (Marschner 1995). On the other hand, selenate is the predominant Se species in well aerated soils. Selenite is adsorbed to soil particles to a much greater extent than selenate, therefore selenate is much more readily taken up by plant roots than selenite (Terry et al. 2000).

A close chemical and physical similarity exists between Se and sulphur (S). Uptake of sulphate by plant roots is mediated by a high affinity permease and selenate competes with sulphate for the binding sites of this permease (White et al. 2004, Sors et al 2005). Therefore, selenate uptake can be strongly decreased by high sulphate supply and the presence of elevated $\mathrm{SO}_{4}^{-2}$ concentrations has been shown to drastically reduce the uptake of Se for many plants including wheat (Grieve et al., 1999) and several varieties of Brassica (Kopsell and Randle 1999, Suarez et al. 2003).

Uptake and accumulation of Se by plants is determined by Se form and concentration and the presence of competing ions (Zayed et al. 1998). Additionally, plant species differ very much in Se uptake and accumulation in the shoots and also in their capacity to tolerate high Se concentrations in the rooting medium or in the shoot tissue or in both. In this regard, plants can be classified into Se-accumulators and non-accumulators, and those between as Se-indicators (Marschner 1995) or secondary Se accumulators (Terry et al. 2000).

Se volatilization is the process by which inorganic Se is converted to volatile forms (Zayed et al. 1998). Dimethylselenides the predominant form of volatile Se, is 500 to 700 times less toxic than inorganic forms of Se (Wilber 1980). Thus it is possible to use plant volatilization as one dispersal pathway for excessive Se concentrations in soils with naturally elevated concentrations of Se, such as those derived from some sedimentary rock or those impacted by anthropogenic inputs.

Although Se is not known to be essential for higher plants, it is an essential trace element in mammalian nutrition (Ellis and Salt 2003). Health benefits associated with Se intake include role in reducing viral infection, improving immune function, slowing the aging process and reducing cardiovascular disease. Se status in a population correlates highly with the Se content of locally produced crops (Combs 1989). In areas of the world with low Se in the soil, Se fertilization is practiced to avoid Se deficiencies in animals and humans (Gissel-Nielsen et al. 1984). Consumption of traditional dietary vegetables would be a good way to deliver organic Se to a population, and ensure that beneficial effects may be realize over the life span of an individual. Positive effects of Se biofortification of food crops on human health were frequently reported (Broadley et al 2006). Several forage Brassica species are important vegetable crops that could be enriched with Se to supplement the human diet or can be used as nutritional supplements to meet the nutritional requirement of livestock (Ekholm 1997, Ellis and Salt 2003).

However, a dietary intake one to two orders of magnitude greater than those leading to Se deficiency may cause chronic toxicity. Plants represent the primary route by which Se enters terrestrial food web, therefore factors that influence the Se uptake by plants may be of considerable importance (Ebbs and Weinstein 2001).

It was reported that, members of Cruciferae have an exceptionally high requirement for $\mathrm{S}$ (Marschner 1995) and simultaneously accumulate relatively large amounts of Se and might contain, and tolerate, several hundred $\mu \mathrm{g} \mathrm{Se} \mathrm{g}^{-1}$ shoot dry matter (Bañuelos et al. 1997). Se uptake and its effect on growth of alfalfa as an important feed crop have been studied by many authors (Soltanpour and Workman 1980, Mikkelsen et al 1987, 1988). It was shown that alfalfa is highly susceptible to Se and toxicity can occur at contents below $100 \mu \mathrm{g} \mathrm{Se} \mathrm{g}^{-1}$ (Shrift 1969). The critical shoot Se concentrations correspond to a $10 \%$ reduction in the yield of some crop species including mustard 
Vol. 17 (2008): 177-190.

and alfalfa grown in the presence of selenate were presented by White (et al 2004).

The main objective of this work was study of the effect of S nutritional status on uptake, accumulation and volatilization of Se. The second aim was to evaluate the beneficial effect of Se on growth of plants particularly in dependence of $\mathrm{S}$ supply. Important feed and food crops including two varieties of Brassica oleracea (cabbage and kohlrabi) and alfalfa were used in this work, because of their importance in Se input to food chain directly to human and/or via animals.

\section{Material and methods}

\section{Plant materials}

Two varieties of Brassica oleracea $\mathrm{L}$. including var. Sabauda (Cabbage) and var. Gongyloides (Kohlrabi) and one local cultivar of alfalfa (Medicago sativa L. cv. Gareh-yondjeh) were used for this study. Seeds were provided by the Agricultural Research Center, Tabriz, Iran.

\section{Plant cultivation and treatments}

Seeds were surface-sterilized using sodiumhypochlorite at $5 \%$, then were germinated in the dark on filter paper (cabbage and kohlrabi) or vermiculite (alfalfa) soaked with saturated $\mathrm{CaSO}_{4}$ solution. Six-day-old young seedlings were precultured for 16 days in 50\% nutrient solution either in hydroponic medium (cabbage and kohlrabi) or on vermiculite (alfalfa). Thereafter, plants were transplanted to 21 dark pots (one plant per pot) and were pre-cultured for another 2 days. After preculture, plants were transferred to treatment solutions with full strength nutrient solution (Hoagland and Arnon 1950) containing one of four levels of $S$ including $0.05,0.25,0.5,1 \mathrm{mM}$ and five levels of Se including $0,5,10,15$ and $20 \mu \mathrm{M}$ (as sodium selenate, $\mathrm{Na}_{2} \mathrm{SeO}_{4} \cdot 10 \mathrm{H}_{2} \mathrm{O}$ ). Application of lower $\mathrm{S}$ treatments than the complete nutrient solution (1.0
$\mathrm{mM}$ ) was realized by change in the concentration of $\mathrm{MgSO}_{4}$ and addition of equi-molar amounts of $\mathrm{MgCl}_{2}$ to the medium. Nutrient solutions were changed completely each 4 days and $\mathrm{pH}$ of nutrient solutions was adjusted each day.

Plants were grown under controlled environmental conditions with a temperature regime of $25^{\circ} / 18^{\circ} \mathrm{C}$ day/night, $14 / 10 \mathrm{~h}$ light/dark period, a relative humidity of $70 / 80 \%$ and at a photon flux density of about $400 \mu \mathrm{mol} \mathrm{m} \mathrm{m}^{-2} \mathrm{~s}^{-1}$.

\section{Plant harvest and analysis}

After growing for 14 days, plants were harvested. Each plant was divided into leaves and root, leaves were washed with double-distilled water and after determining fresh weight, leaf area was measured using a planometer. Roots were washed with double-distilled water for $5 \mathrm{~min}$ and after blotting dry, fresh weight and root length (Tennant 1975) were determined.

After drying at $70{ }^{\circ} \mathrm{C}$ for 1 day to determine dry weight (DW), samples were digested in perchloric acid $(1: 15 \mathrm{w} / \mathrm{v})$ on heating plate for 5-6 h. Thereafter, digested samples were re-suspended in $1 \% \mathrm{HCl}$ and made to volume by double-distilled water.

$\mathrm{S}$ analysis was carried out according to turbidity method (Gupta 1999). In an aliquot of the sample solution, $\mathrm{BaSO}_{4}$ turbidity was developed in the presence of sodium chloride / hydrochloric acid buffer, ethanol and glycerol by dissolving $\mathrm{BaCl}_{2}$ crystals. The absorbance of the $\mathrm{BaSO}_{4}$ turbidity was measured at $420 \mathrm{~nm}$ by spectrophotometer (Specord 200, Analytical Jena, Germany). The content of $\mathrm{S}$ was estimated by reference to the standard calibration curve obtained for $\mathrm{K}_{2} \mathrm{SO}_{4}$. Se was determined by atomic absorption spectrometry (Shimadzu, AA 6500) with an automated hydride generation accessory (HVG-1). The hydride generator was operated under the conditions of $6.5,1.0$ and $1.0 \mathrm{ml} \mathrm{min}-1$ flow rate for sample, $\mathrm{NaBH}_{4}(0.6 \% \mathrm{w} / \mathrm{v}$ in $0.5 \% \mathrm{w} / \mathrm{v} \mathrm{NaOH})$ and $\mathrm{HCl}(10 \mathrm{M})$ respectively and nitrogen pressure of $350 \mathrm{KPa}$. The AA spectrometer was set at the wavelength of $196 \mathrm{~nm}$ (Norheim and Haugen 
Hajiboland, R. \& Amjad, L. Accumulation and volatilization of selenium in two Brassica varieties

1986). Series of Se standard solutions consisting of 5 different concentrations ranging from 0 to 40 $\mu \mathrm{g} 1^{-1}$ were prepared by heating sodium selenate $(\mathrm{Se}(\mathrm{VI}))$ in the presence of $25 \%$ (v/v) hydrochloric acid until reduction to selenite (Se(IV)) was complete (Hobbins 1981).

\section{Collection and measurement of volatile $\mathrm{Se}$}

Plants grown for 14 days at adequate $\mathrm{S}$ supply ( $\mathrm{S}$ $=1.0 \mathrm{mM})$ and supplemented with three levels of Se $(0,10$ and $20 \mu \mathrm{M})$ as described above, were used for study of Se volatilization. A transparent acrylic plastic (Plexiglas) chamber was built to capture Se volatilized from plants. The chamber was $0.3 \times 0.3 \mathrm{~m}$ in horizontal cross section and 0.5 $\mathrm{m}$ in height. Plants, which have been treated for 14 days, were placed in the chamber for $24 \mathrm{~h}$ under temperature between $25-28^{\circ} \mathrm{C}$ and illumination of $300 \mu \mathrm{mol} \mathrm{m} \mathrm{m}^{-2} \mathrm{~s}^{-1}$. One gram of activated carbon (Sigma) was placed in the chamber and volatile Se captured on the activated carbon and extracted by washing with water. The concentration of Se in the wash water was analyzed by atomic absorption following reduction and hydride generation (Lewis et al. 1966).

$\mathrm{S}$ and Se uptake and transport were calculated on the basis of root DW (Marschner, 1995) according to the following formulae and were expressed as (mg or $\mu \mathrm{g} \mathrm{g}^{-1}$ root DW):

\section{Uptake $=($ Total S $(\mathrm{Se})$ content of shoot and root $)$ / root $D W$ \\ Transport $=$ Content of $S(\mathrm{Se})$ in shoot / root $D W$.}

All required chemicals were purchased from Merck and Fluka chemical companies with pro analysis grade or higher. Experiments were carried out in completely randomized block design with 4 replications. One-way ANOVA followed by Tukey test $(p<0.05)$ for multiple comparisons was used and performed using Sigma Stat 2.03.

\section{Results}

\section{Effect of Se on plants growth and dry matter production}

Se affected growth differently depending on S supply level. At very low $\mathrm{S}$ supply $(0.05 \mathrm{mM}$ in cabbage and $0.25 \mathrm{mM}$ in alfalfa), Se inhibited plants growth at all Se concentrations and for all measured growth parameters. In alfalfa, the toxicity effect of Se at very low $\mathrm{S}$ supply $(0.05 \mathrm{mM})$ was very high, so that, plants died in the early growth period and did not remain alive until harvest. However, in kohlrabi supplied by very low $(0.05 \mathrm{mM}) \mathrm{S}$, in the presence of $5 \mu \mathrm{M}$ Se rather an increase in shoot and root DW was observed (Table 1).

The most sensitive parameter to Se toxicity in cabbage and kohlrabi was leaf area, which reduced $82 \%$ and $74 \%$ in cabbage (Table 1) and kohlrabi (Table 2) in response to $20 \mu \mathrm{M}$ Se respectively. Shoot DW was affected by Se toxicity at $45 \%$ and $50 \%$ under similar Se concentration in cabbage and kohlrabi respectively.

A significant growth improvement was observed at Se concentration of 5 and $10 \mu \mathrm{M}$ when plants were supplied with 0.25 and $0.5 \mathrm{mM} \mathrm{S}$, and at Se concentration of 15 and $20 \mu \mathrm{M}$ when plants were supplied with 0.5 and $1.0 \mathrm{mM} \mathrm{S}$. The highest growth improvement was observed in cabbage with up to $59 \%$ increase in shoot DW and $55 \%$ in leaf area. The growth promoting effect of Se was much lower pronounced in kohlrabi and particularly in alfalfa than in cabbage. Only in S sufficient plants $(1.0 \mathrm{mM})$ an increase in shoot DW (35\%) was observed in response to $20 \mu \mathrm{M}$ Se in kohlrabi and alfalfa. Changes in other growth parameters such as leaf area were rather in tendency than significant (Table 3).

\section{$S$ and Se concentration of shoot and roots}

Shoot and root concentration of S was not drastically affected by Se addition. However, a signifi- 
Vol. 17 (2008): 177-190.

Table 1. Effect of various concentrations of Se on shoot and root dry weight (DW), root length and leaf area at different nutritional status of S in cabbage (Brassica oleracea L. var. Sabauda). Data are mean \pm SD. Values in each column within each $\mathrm{S}$ treatment followed by the same letter are not significantly different $(p<0.05)$.

\begin{tabular}{|c|c|c|c|c|c|}
\hline $\begin{array}{c}\mathrm{S} \\
(\mathrm{mM})\end{array}$ & $\begin{array}{c}\mathrm{Se} \\
(\mu \mathrm{M})\end{array}$ & $\begin{array}{c}\text { Shoot DW } \\
\text { (mg per plant) }\end{array}$ & $\begin{array}{c}\text { Root DW } \\
\text { (mg per plant) }\end{array}$ & $\begin{array}{l}\text { Root length } \\
\text { (cm per plant) }\end{array}$ & $\begin{array}{c}\text { Leaf area } \\
\left(\mathrm{cm}^{2} \text { per plant }\right)\end{array}$ \\
\hline \multirow[t]{5}{*}{0.05} & 0 & $175 \pm 4^{\mathrm{a}}$ & $18 \pm 3^{\mathrm{a}}$ & $185 \pm 39^{a}$ & $50 \pm 8^{a}$ \\
\hline & 5 & $77 \pm 18^{c}$ & $7 \pm 3^{b}$ & $29 \pm 8^{b}$ & $13 \pm 5^{b}$ \\
\hline & 10 & $108 \pm 25^{\mathrm{cb}}$ & $9 \pm 5^{b}$ & $38 \pm 9^{\mathrm{b}}$ & $14 \pm 8^{b}$ \\
\hline & 15 & $122 \pm 24^{b}$ & $7 \pm 3^{b}$ & $29 \pm 7^{b}$ & $11 \pm 5^{\mathrm{b}}$ \\
\hline & 20 & $96 \pm 19$ cb & $6 \pm 2 \mathrm{~b}$ & $31 \pm 8^{b}$ & $9 \pm 3^{b}$ \\
\hline \multirow[t]{5}{*}{0.25} & 0 & $473 \pm 30^{b}$ & $38 \pm 8$ bc & $265 \pm 37^{b}$ & $142 \pm 27^{b}$ \\
\hline & 5 & $737 \pm 63^{\mathrm{a}}$ & $49 \pm 4$ ab & $427 \pm 57^{\mathrm{a}}$ & $203 \pm 32^{\mathrm{a}}$ \\
\hline & 10 & $682 \pm 59^{\text {a }}$ & $64 \pm 14^{\text {a }}$ & $301 \pm 32 \mathrm{ab}$ & $176 \pm 32^{a b}$ \\
\hline & 15 & $425 \pm 63^{b}$ & $36 \pm 12 \mathrm{bc}$ & $159 \pm 33^{c}$ & $79 \pm 12^{c}$ \\
\hline & 20 & $350 \pm 20^{b}$ & $27 \pm 6^{c}$ & $181 \pm 28^{c}$ & $58 \pm 11^{\mathrm{c}}$ \\
\hline \multirow[t]{5}{*}{0.5} & 0 & $648 \pm 37^{b}$ & $51 \pm 14^{\text {a }}$ & $165 \pm 23^{c}$ & $182 \pm 15$ ab \\
\hline & 5 & $555 \pm 29^{c}$ & $46 \pm 13^{a}$ & $309 \pm 20^{a}$ & $161 \pm 23 \mathrm{ab}$ \\
\hline & 10 & $756 \pm 34^{\mathrm{a}}$ & $67 \pm 12^{a}$ & $297 \pm 19^{b}$ & $218 \pm 27^{\mathrm{a}}$ \\
\hline & 15 & $642 \pm 50 \mathrm{bc}$ & $52 \pm 16^{\mathrm{a}}$ & $189 \pm 22^{\mathrm{c}}$ & $137 \pm 23^{b}$ \\
\hline & 20 & $651 \pm 51^{\mathrm{b}}$ & $44 \pm 14^{\text {a }}$ & $268 \pm 19^{b}$ & $153 \pm 27^{b}$ \\
\hline \multirow[t]{5}{*}{1.0} & 0 & $665 \pm 57^{b}$ & $56 \pm 3$ ab & $246 \pm 23^{c}$ & $196 \pm 27^{b}$ \\
\hline & 5 & $499 \pm 29^{c}$ & $42 \pm 4^{b}$ & $541 \pm 38^{a}$ & $156 \pm 21^{b}$ \\
\hline & 10 & $684 \pm 36^{b}$ & $38 \pm 12^{b}$ & $270 \pm 19^{c}$ & $193 \pm 23^{b}$ \\
\hline & 15 & $1056 \pm 67^{\text {a }}$ & $80 \pm 18^{\mathrm{a}}$ & $262 \pm 30^{c}$ & $304 \pm 42^{\text {a }}$ \\
\hline & 20 & $962 \pm 40^{\mathrm{a}}$ & $61 \pm 19^{a b}$ & $354 \pm 14^{b}$ & $273 \pm 21^{\mathrm{a}}$ \\
\hline
\end{tabular}

Table 2. Effect of various concentrations of Se on shoot and root dry weight (DW), root length and leaf area at different nutritional status of S in Kohlrabi (Brassica oleracea L. var. Gongyloides). Data are mean \pm SD. Values in each column within each $\mathrm{S}$ treatment followed by the same letter are not significantly different $(p<0.05)$.

\begin{tabular}{|c|c|c|c|c|c|}
\hline $\begin{array}{c}\mathrm{S} \\
(\mathrm{mM})\end{array}$ & $\begin{array}{c}\mathrm{Se} \\
(\mu \mathrm{M})\end{array}$ & $\begin{array}{c}\text { Shoot DW } \\
\text { (mg per plant) }\end{array}$ & $\begin{array}{c}\text { Root DW } \\
\text { (mg per plant) }\end{array}$ & $\begin{array}{l}\text { Root length } \\
\text { (cm per plant) }\end{array}$ & $\begin{array}{c}\text { Leaf area } \\
\left(\mathrm{cm}^{2} \text { per plant }\right)\end{array}$ \\
\hline \multirow[t]{5}{*}{0.05} & 0 & $442 \pm 29^{\mathrm{b}}$ & $33 \pm 9^{\mathrm{b}}$ & $250 \pm 10^{\mathrm{a}}$ & $102 \pm 32^{a}$ \\
\hline & 5 & $582 \pm 31^{a}$ & $54 \pm 11^{a}$ & $76 \pm 11^{\mathrm{c}}$ & $83 \pm 10^{a b}$ \\
\hline & 10 & $272 \pm 39^{c}$ & $17 \pm 8^{b}$ & $81 \pm 17^{\mathrm{c}}$ & $31 \pm 8^{c}$ \\
\hline & 15 & $276 \pm 38^{c}$ & $21 \pm 8^{b}$ & $190 \pm 39^{\mathrm{a}}$ & $45 \pm 5^{c}$ \\
\hline & 20 & $221 \pm 21^{\mathrm{c}}$ & $14 \pm 7^{b}$ & $105 \pm 5^{b}$ & $26 \pm 3^{d}$ \\
\hline \multirow[t]{5}{*}{0.25} & 0 & $732 \pm 37^{\mathrm{a}}$ & $60 \pm 14^{\text {a }}$ & $304 \pm 23^{b}$ & $163 \pm 28^{a}$ \\
\hline & 5 & $724 \pm 30^{a}$ & $76 \pm 17^{a}$ & $537 \pm 23^{a}$ & $174 \pm 11^{\mathrm{a}}$ \\
\hline & 10 & $461 \pm 34^{c}$ & $50 \pm 19^{a}$ & $148 \pm 22^{c}$ & $79 \pm 13^{b}$ \\
\hline & 15 & $444 \pm 39^{c}$ & $48 \pm 12^{a}$ & $292 \pm 16^{b}$ & $81 \pm 15^{b}$ \\
\hline & 20 & $651 \pm 43^{b}$ & $48 \pm 18^{a}$ & $313 \pm 20^{b}$ & $140 \pm 25^{\mathrm{a}}$ \\
\hline \multirow[t]{5}{*}{0.5} & 0 & $641 \pm 36^{b}$ & $56 \pm 18^{a}$ & $471 \pm 19^{b}$ & $149 \pm 31 \mathrm{ab}$ \\
\hline & 5 & $729 \pm 47^{\mathrm{a}}$ & $65 \pm 16^{a}$ & $275 \pm 27^{\mathrm{c}}$ & $215 \pm 32^{a}$ \\
\hline & 10 & $758 \pm 49^{\text {a }}$ & $61 \pm 17^{a}$ & $174 \pm 28^{d}$ & $179 \pm 44^{\mathrm{a}}$ \\
\hline & 15 & $467 \pm 40^{c}$ & $41 \pm 13^{a}$ & $570 \pm 38^{a}$ & $100 \pm 27^{b}$ \\
\hline & 20 & $569 \pm 33^{b}$ & $46 \pm 10^{a}$ & $258 \pm 28^{\mathrm{c}}$ & $128 \pm 23^{b}$ \\
\hline \multirow[t]{5}{*}{1.0} & 0 & $599 \pm 36^{b}$ & $50 \pm 5^{a}$ & $322 \pm 24^{c}$ & $147 \pm 6^{\mathrm{a}}$ \\
\hline & 5 & $632 \pm 42^{b}$ & $62 \pm 13^{a}$ & $391 \pm 25 \mathrm{ab}$ & $186 \pm 17^{\mathrm{a}}$ \\
\hline & 10 & $577 \pm 54^{b}$ & $53 \pm 10^{a}$ & $311 \pm 14^{\mathrm{c}}$ & $157 \pm 22^{\mathrm{a}}$ \\
\hline & 15 & $808 \pm 53^{a}$ & $60 \pm 13^{a}$ & $351 \pm 29 \mathrm{bc}$ & $173 \pm 38^{\mathrm{a}}$ \\
\hline & 20 & $747 \pm 39^{\text {a }}$ & $60 \pm 12^{a}$ & $487 \pm 35^{\text {a }}$ & $183 \pm 24^{\mathrm{a}}$ \\
\hline
\end{tabular}


Hajiboland, R. \& Amjad, L. Accumulation and volatilization of selenium in two Brassica varieties

Table 3. Effect of various concentrations of Se on shoot and root dry weight (DW), root length and leaf area at different nutritional status of S in alfalfa (Medicago sativa L. cv. Garehyounjeh). Data are mean \pm SD. Values in each column within each $\mathrm{S}$ treatment followed by the same letter are not significantly different $(p<0.05)$.

\begin{tabular}{|c|c|c|c|c|c|}
\hline $\begin{array}{c}\mathrm{S} \\
(\mathrm{mM})\end{array}$ & $\begin{array}{c}\mathrm{Se} \\
(\mu \mathrm{M})\end{array}$ & $\begin{array}{c}\text { Shoot DW } \\
\text { (mg per plant) }\end{array}$ & $\begin{array}{c}\text { Root DW } \\
\text { (mg per plant) }\end{array}$ & $\begin{array}{l}\text { Root length } \\
\text { (cm per plant) }\end{array}$ & $\begin{array}{c}\text { Leaf area } \\
\left(\mathrm{cm}^{2} \text { per plant }\right)\end{array}$ \\
\hline \multirow[t]{5}{*}{0.05} & 0 & $120 \pm 35$ & $25 \pm 6$ & $246 \pm 53$ & $18 \pm 4$ \\
\hline & 5 & died & died & died & died \\
\hline & 10 & died & died & died & died \\
\hline & 15 & died & died & died & died \\
\hline & 20 & died & died & died & died \\
\hline \multirow[t]{5}{*}{0.25} & 0 & $126 \pm 10^{a}$ & $17 \pm 3^{\text {a }}$ & $243 \pm 32^{\mathrm{a}}$ & $15 \pm 1^{a}$ \\
\hline & 5 & $101 \pm 9^{\text {a }}$ & $13 \pm 5^{\mathrm{a}}$ & $246 \pm 30^{a}$ & $15 \pm 3^{\text {a }}$ \\
\hline & 10 & $127 \pm 15^{a}$ & $14 \pm 5^{\text {a }}$ & $254 \pm 24^{\mathrm{a}}$ & $18 \pm 7$ a \\
\hline & 15 & $131 \pm 21^{a}$ & $15 \pm 4^{\mathrm{a}}$ & $253 \pm 7^{\mathrm{a}}$ & $17 \pm 3^{a}$ \\
\hline & 20 & $128 \pm 15^{a}$ & $16 \pm 3^{a}$ & $251 \pm 22^{a}$ & $15 \pm 3^{\text {a }}$ \\
\hline \multirow[t]{5}{*}{0.5} & 0 & $143 \pm 21^{a}$ & $24 \pm 2^{a}$ & $276 \pm 53^{a}$ & $18 \pm 2^{\mathrm{a}}$ \\
\hline & 5 & $104 \pm 17^{\mathrm{a}}$ & $14 \pm 5^{b}$ & $223 \pm 45^{\text {a }}$ & $13 \pm 1^{a}$ \\
\hline & 10 & $150 \pm 21^{a}$ & $18 \pm 2^{a b}$ & $203 \pm 35^{\text {a }}$ & $20 \pm 6^{\text {a }}$ \\
\hline & 15 & $185 \pm 71^{a}$ & $20 \pm 5^{\text {a }}$ & $237 \pm 66^{a}$ & $21 \pm 5^{\mathrm{a}}$ \\
\hline & 20 & $157 \pm 44^{\mathrm{a}}$ & $16 \pm 2^{b}$ & $179 \pm 38^{\mathrm{a}}$ & $17 \pm 2^{\mathrm{a}}$ \\
\hline \multirow[t]{5}{*}{1.0} & 0 & $142 \pm 9^{b}$ & $17 \pm 1^{\mathrm{a}}$ & $317 \pm 32^{a b}$ & $17 \pm 2^{\mathrm{a}}$ \\
\hline & 5 & $171 \pm 25 \mathrm{ab}$ & $15 \pm 5^{\text {a }}$ & $358 \pm 52^{\mathrm{a}}$ & $25 \pm 6^{\mathrm{a}}$ \\
\hline & 10 & $134 \pm 20^{b}$ & $19 \pm 5^{\text {a }}$ & $222 \pm 46^{b}$ & $18 \pm 8^{\mathrm{a}}$ \\
\hline & 15 & $178 \pm 29$ ab & $19 \pm 2^{\mathrm{a}}$ & $299 \pm 77$ ab & $19 \pm 4^{\mathrm{a}}$ \\
\hline & 20 & $202 \pm 14^{\mathrm{a}}$ & $19 \pm 2^{\mathrm{a}}$ & $265 \pm 70^{a b}$ & $25 \pm 6^{\mathrm{a}}$ \\
\hline
\end{tabular}

cant enhancement of $\mathrm{S}$ concentration which was reduced again in response to increasing Se levels in the medium was observed. Such an effect tended to be more pronounced in $\mathrm{S}$ deficient plants. As expected, accumulation of Se responded strongly to Se addition. In some $\mathrm{S} / \mathrm{Se}$ treatment combinations, however, Se was not accumulated continuously in response to increasing Se levels. After the first increase, a significant reduction was observed followed usually by a further increase in Se accumulation (Table 4).

\section{Effect of Se addition on $S$ uptake and transport}

$\mathrm{S}$ uptake and transport increased in response to Se treatment from 5 to $20 \mu \mathrm{M}$. However, at some $\mathrm{S}$ and Se treatment combinations, both uptake and transport decreased with continuing increase of Se concentration particularly in cabbage. However, comparing Se treatment of 0 and $20 \mu \mathrm{M}$, a significant enhancing effect of Se on S uptake and transport could be demonstrated in all three studied species/ varieties. The proportion of transported $\mathrm{S}$ into shoot changed in response to Se supply, so that, this proportion was increased in the presence of Se, but the effect of different Se concentrations were similar. However, such an effect of Se on proportion of S transport was not observed in alfalfa (Table 5).

\section{Se uptake, transport and volatilization}

Se uptake increased with increasing Se concentration in the medium. However, in two varieties of Brassica supplied with very low $\mathrm{S}(0.05 \mathrm{mM})$, Se uptake did not change in response to Se addition from 5 to 20 
Vol. 17 (2008): 177-190.

Table 4. Concentration of S ( $\left.\mathrm{mg} \mathrm{g}^{-1} \mathrm{DW}\right)$ and $\mathrm{Se}\left(\mu \mathrm{g} \mathrm{g} \mathrm{g}^{-1} \mathrm{DW}\right)$ in shoot and roots of cabbage (Brassica oleracea L. var. Sabauda), kohlrabi (Brassica oleracea L. var. Gongyloides) and alfalfa (Medicago sativa L. cv. Garehyounjeh) grown at different levels of $\mathrm{S}$ and $\mathrm{Se}$ in the medium. Data are mean $\pm \mathrm{SD}$. Values in each column within each $\mathrm{S}$ treatment followed by the same letter are not significantly different $(p<0.05)$.

\begin{tabular}{|c|c|c|c|c|c|c|c|}
\hline \multirow{3}{*}{$\begin{array}{c}\mathrm{S} \\
(\mathrm{mM})\end{array}$} & \multirow{3}{*}{$\begin{array}{c}\mathrm{Se} \\
(\mu \mathrm{M})\end{array}$} & \multicolumn{2}{|c|}{ Cabbage } & \multicolumn{2}{|c|}{ Kohlrabi } & \multicolumn{2}{|c|}{ Alfalfa } \\
\hline & & $\mathrm{S}$ & $\mathrm{Se}$ & $S$ & $\mathrm{Se}$ & $\mathrm{S}$ & $\mathrm{Se}$ \\
\hline & & \multicolumn{6}{|c|}{ Shoot } \\
\hline \multirow[t]{5}{*}{0.05} & 0 & $9.7 \pm 1.4^{\mathrm{ab}}$ & $2 \pm 0.4^{c}$ & $3.7 \pm 0.9^{b}$ & $20 \pm 4^{\mathrm{e}}$ & $1.2 \pm 0.1$ & $8 \pm 2$ \\
\hline & 5 & $12.5 \pm 0.6^{\mathrm{a}}$ & $2148 \pm 131^{\mathrm{a}}$ & $2.6 \pm 0.6^{b}$ & $765 \pm 144^{d}$ & --- & --- \\
\hline & 10 & $6.4 \pm 0.5^{\mathrm{b}}$ & $1129 \pm 190^{b}$ & $4.9 \pm 1.7^{\mathrm{a}}$ & $4476 \pm 384^{b}$ & --- & --- \\
\hline & 15 & $9.5 \pm 5.1 \mathrm{ab}$ & $982 \pm 109^{b}$ & $2.6 \pm 0.6^{b}$ & $1503 \pm 211^{\mathrm{c}}$ & --- & --- \\
\hline & 20 & $5.5 \pm 1.9^{b}$ & $1002 \pm 146^{b}$ & $2.6 \pm 0.8^{b}$ & $9054 \pm 627^{\mathrm{a}}$ & --- & --- \\
\hline \multirow[t]{5}{*}{0.25} & 0 & $15.9 \pm 4.0^{\mathrm{a}}$ & $17 \pm 5^{b}$ & $5.9 \pm 1.2^{\mathrm{c}}$ & $15 \pm 1^{\mathrm{b}}$ & $3.0 \pm 0.6^{c}$ & $4 \pm 0.6^{\mathrm{e}}$ \\
\hline & 5 & $14.1 \pm 0.7^{\mathrm{a}}$ & $970 \pm 246^{\mathrm{a}}$ & $9.2 \pm 1.2^{b}$ & $889 \pm 124^{a}$ & $5.3 \pm 0.7^{b}$ & $643 \pm 138^{d}$ \\
\hline & 10 & $15.2 \pm 1.2^{\mathrm{a}}$ & $96 \pm 11^{\mathrm{b}}$ & $9.2 \pm 1.7^{\mathrm{b}}$ & $1092 \pm 173^{a}$ & $5.3 \pm 0.3^{b}$ & $1627 \pm 312^{c}$ \\
\hline & 15 & $18.5 \pm 1.0^{\mathrm{a}}$ & $141 \pm 52^{b}$ & $9.9 \pm 1.8^{\mathrm{b}}$ & $1003 \pm 171^{\mathrm{a}}$ & $10.9 \pm 0.4^{\mathrm{a}}$ & $3123 \pm 461^{b}$ \\
\hline & 20 & $17.7 \pm 4.9^{\mathrm{a}}$ & $911 \pm 175^{a}$ & $14.8 \pm 0.2^{\mathrm{a}}$ & $957 \pm 119^{\mathrm{a}}$ & $11.1 \pm 0.7^{\mathrm{a}}$ & $4316 \pm 267^{a}$ \\
\hline \multirow[t]{5}{*}{0.5} & 0 & $19.8 \pm 4.2^{\mathrm{a}}$ & $34 \pm 13^{\mathrm{d}}$ & $11.1 \pm 0.5^{\mathrm{b}}$ & $13 \pm 2^{\mathrm{d}}$ & $2.4 \pm 0.5^{\mathrm{e}}$ & $4 \pm 0.7^{\mathrm{e}}$ \\
\hline & 5 & $25.4 \pm 3.9^{\mathrm{a}}$ & $305 \pm 72^{c}$ & $14.6 \pm 2.7^{\mathrm{ab}}$ & $113 \pm 27^{\mathrm{c}}$ & $4.4 \pm 0.3^{\mathrm{d}}$ & $352 \pm 32^{d}$ \\
\hline & 10 & $19.9 \pm 3.7^{\mathrm{a}}$ & $319 \pm 40^{\mathrm{c}}$ & $15.3 \pm 1.9^{\mathrm{a}}$ & $239 \pm 39^{b}$ & $5.9 \pm 1.0^{\mathrm{c}}$ & $979 \pm 49^{c}$ \\
\hline & 15 & $20.9 \pm 6.7^{\text {a }}$ & $709 \pm 97^{\mathrm{a}}$ & $15.2 \pm 0.8^{\mathrm{a}}$ & $272 \pm 77^{b}$ & $10.1 \pm 0.1^{\mathrm{a}}$ & $1551 \pm 460^{b}$ \\
\hline & 20 & $23.3 \pm 0.9^{\mathrm{a}}$ & $549 \pm 86^{b}$ & $19.9 \pm 1.4^{\mathrm{a}}$ & $853 \pm 70^{\mathrm{a}}$ & $8.7 \pm 0.8^{\mathrm{b}}$ & $2209 \pm 244^{\mathrm{a}}$ \\
\hline \multirow[t]{6}{*}{1.0} & 0 & $17.6 \pm 3.2^{\mathrm{ab}}$ & $52 \pm 31^{\mathrm{c}}$ & $13.8 \pm 0.4^{\mathrm{b}}$ & $12 \pm 1^{\mathrm{d}}$ & $2.8 \pm 0.7^{b}$ & $24 \pm 5^{\mathrm{d}}$ \\
\hline & 5 & $29.7 \pm 0.5^{\mathrm{a}}$ & $182 \pm 34^{b}$ & $13.6 \pm 1.8^{\mathrm{b}}$ & $115 \pm 18^{\mathrm{c}}$ & $3.8 \pm 0.7^{b}$ & $330 \pm 54^{b}$ \\
\hline & 10 & $22.2 \pm 1.9^{\mathrm{ab}}$ & $211 \pm 28^{b}$ & $17.7 \pm 3.4^{\mathrm{ab}}$ & $1565 \pm 273^{a}$ & $3.7 \pm 0.7^{b}$ & $247 \pm 52^{b}$ \\
\hline & 15 & $14.5 \pm 10.5^{b}$ & $235 \pm 33^{b}$ & $17.5 \pm 1.9^{\mathrm{ab}}$ & $1259 \pm 232^{\mathrm{a}}$ & $5.2 \pm 0.7^{\mathrm{a}}$ & $116 \pm 37^{c}$ \\
\hline & 20 & $16.4 \pm 10.7^{\mathrm{ab}}$ & $446 \pm 67^{\text {a }}$ & $21.2 \pm 2.4^{\mathrm{a}}$ & $771 \pm 14^{\mathrm{b}}$ & $4.9 \pm 1.2^{\mathrm{a}}$ & $883 \pm 167^{\text {a }}$ \\
\hline & & \multicolumn{6}{|c|}{ Roots } \\
\hline \multirow[t]{5}{*}{0.05} & 0 & $22.9 \pm 2.1^{b}$ & $10 \pm 2^{\mathrm{d}}$ & $13.5 \pm 3.6^{\mathrm{a}}$ & $7 \pm 1^{d}$ & $4.6 \pm 0.9$ & $30 \pm 4$ \\
\hline & 5 & $53.3 \pm 9.9^{b}$ & $7640 \pm 945^{\mathrm{a}}$ & $9.7 \pm 2.1^{\mathrm{a}}$ & $1045 \pm 77^{b}$ & --- & --- \\
\hline & 10 & $30.9 \pm 6.8^{b}$ & $4952 \pm 80^{\mathrm{b}}$ & $28.2 \pm 14.2^{\mathrm{a}}$ & $543 \pm 293^{\mathrm{c}}$ & --- & --- \\
\hline & 15 & $57.5 \pm 24.8^{b}$ & $2885 \pm 649^{c}$ & $19.6 \pm 11.2^{\mathrm{a}}$ & $1410 \pm 118^{a}$ & --- & --- \\
\hline & 20 & $128.6 \pm 37.5^{\mathrm{a}}$ & $6359 \pm 771^{\mathrm{a}}$ & $24.8 \pm 11.6^{\mathrm{a}}$ & $817 \pm 34^{b}$ & --- & --- \\
\hline \multirow[t]{5}{*}{0.25} & 0 & $9.3 \pm 3.7^{\mathrm{b}}$ & $22 \pm 8^{\mathrm{e}}$ & $9.4 \pm 1.2^{\mathrm{a}}$ & $24 \pm 4^{c}$ & $7.6 \pm 0.9^{b}$ & $20 \pm 3^{\mathrm{d}}$ \\
\hline & 5 & $12.2 \pm 1.4^{\mathrm{ab}}$ & $1580 \pm 237^{\mathrm{d}}$ & $3.8 \pm 0.3^{b}$ & $671 \pm 18^{\mathrm{a}}$ & $7.2 \pm 1.9^{b}$ & $788 \pm 114^{c}$ \\
\hline & 10 & $9.8 \pm 0.2^{b}$ & $2611 \pm 280^{c}$ & $7.9 \pm 2.8^{\mathrm{a}}$ & $290 \pm 30^{b}$ & $9.4 \pm 1.3^{\mathrm{ab}}$ & $1284 \pm 233^{c}$ \\
\hline & 15 & $15.5 \pm 3.3^{\mathrm{a}}$ & $4487 \pm 526^{b}$ & $8.4 \pm 1.9^{\mathrm{a}}$ & $558 \pm 102^{a}$ & $6.9 \pm 0.7^{b}$ & $4021 \pm 224^{b}$ \\
\hline & 20 & $9.9 \pm 0.7^{\mathrm{b}}$ & $6633 \pm 491^{\mathrm{a}}$ & $5.8 \pm 1.3^{\mathrm{ab}}$ & $394 \pm 42^{b}$ & $11.9 \pm 2.0^{\mathrm{a}}$ & $5760 \pm 788^{a}$ \\
\hline \multirow[t]{5}{*}{0.5} & 0 & $11.0 \pm 3.5^{\mathrm{ab}}$ & $32 \pm 8^{\mathrm{d}}$ & $9.4 \pm 1.4^{\mathrm{a}}$ & $30 \pm 4^{c}$ & $11.4 \pm 0.2^{\mathrm{a}}$ & $33 \pm 5^{\mathrm{e}}$ \\
\hline & 5 & $14.6 \pm 1.8^{\mathrm{a}}$ & $870 \pm 191^{\mathrm{c}}$ & $6.7 \pm 1.6^{\mathrm{a}}$ & $1120 \pm 164^{\mathrm{a}}$ & $7.8 \pm 2.3^{\mathrm{ab}}$ & $578 \pm 95^{\mathrm{d}}$ \\
\hline & 10 & $6.9 \pm 1.9^{\mathrm{b}}$ & $1884 \pm 380^{b}$ & $4.4 \pm 0.4^{\mathrm{a}}$ & $272 \pm 63^{b}$ & $6.8 \pm 1.3^{b}$ & $1111 \pm 152^{\mathrm{c}}$ \\
\hline & 15 & $9.3 \pm 3.0^{\mathrm{ab}}$ & $2933 \pm 510^{a}$ & $8.5 \pm 7.0^{\mathrm{a}}$ & $896 \pm 194^{\mathrm{a}}$ & $6.1 \pm 0.9^{b}$ & $1524 \pm 196^{b}$ \\
\hline & 20 & $12.3 \pm 0.38^{\mathrm{ab}}$ & $2868 \pm 405^{a}$ & $14.4 \pm 7.2^{\mathrm{a}}$ & $1080 \pm 126^{a}$ & $9.9 \pm 2.8^{\mathrm{ab}}$ & $2044 \pm 115^{a}$ \\
\hline \multirow[t]{5}{*}{1.0} & 0 & $10.8 \pm 0.4 \mathrm{bc}$ & $25 \pm 4^{\mathrm{c}}$ & $10.1 \pm 1.6^{\mathrm{a}}$ & $27 \pm 5^{c}$ & $14.8 \pm 2.7^{\mathrm{ab}}$ & $30 \pm 7^{c}$ \\
\hline & 5 & $16.4 \pm 2.1^{\mathrm{a}}$ & $446 \pm 92^{b}$ & $8.4 \pm 1.5^{\mathrm{a}}$ & $83 \pm 23^{c}$ & $8.4 \pm 1.1^{\mathrm{b}}$ & $229 \pm 58^{b}$ \\
\hline & 10 & $11.8 \pm 2.8^{b}$ & $726 \pm 161^{b}$ & $6.0 \pm 0.8^{\mathrm{a}}$ & $855 \pm 108^{a}$ & $9.5 \pm 3.2^{b}$ & $434 \pm 82^{\mathrm{b}}$ \\
\hline & 15 & $7.2 \pm 2.4^{\mathrm{c}}$ & $1316 \pm 204^{\mathrm{a}}$ & $6.1 \pm 1.0^{\mathrm{a}}$ & $797 \pm 168^{a}$ & $11.4 \pm 2.9^{\mathrm{ab}}$ & $1115 \pm 209^{a}$ \\
\hline & 20 & $11.2 \pm 0.5^{b c}$ & $1368 \pm 191^{\mathrm{a}}$ & $9.1 \pm 3.6^{\mathrm{a}}$ & $512 \pm 92^{b}$ & $19.3 \pm 6.2^{\mathrm{a}}$ & $895 \pm 68^{\mathrm{a}}$ \\
\hline
\end{tabular}


Hajiboland, R. \& Amjad, L. Accumulation and volatilization of selenium in two Brassica varieties

Table 5. Effect of various concentrations of Se on uptake and transport of S ( $\mathrm{mg} \mathrm{g}^{-1}$ root dry weight, (DW)) at different S nutritional status in cabbage (Brassica oleracea L. var. Sabauda), kohlrabi (Brassica oleracea L. var. Gongyloides) and alfalfa (Medicago sativa L. cv. Garehyounjeh). Data are mean \pm SD. Values in each column within each $\mathrm{S}$ treatment followed by the same letter are not significantly different $(p<0.05)$.

\begin{tabular}{|c|c|c|c|c|c|c|c|}
\hline \multirow{2}{*}{$\begin{array}{c}\mathrm{S} \\
(\mathrm{mM})\end{array}$} & \multirow{2}{*}{$\begin{array}{c}\mathrm{Se} \\
(\mu \mathrm{M})\end{array}$} & \multicolumn{2}{|c|}{ Cabbage } & \multicolumn{2}{|c|}{ Kohlrabi } & \multicolumn{2}{|c|}{ Alfalfa } \\
\hline & & Uptake & Transport & Uptake & Transport & Uptake & Transport \\
\hline \multirow[t]{5}{*}{0.05} & 0 & $118 \pm 4^{\text {a }}$ & $96 \pm 2^{\mathrm{ab}}$ & $64 \pm 18^{b}$ & $50 \pm 14^{\mathrm{ab}}$ & $11 \pm 1$ & $6 \pm 0.4$ \\
\hline & 5 & $185 \pm 16^{\mathrm{a}}$ & $131 \pm 6^{\mathrm{ab}}$ & $37 \pm 7^{b}$ & $28 \pm 5^{b}$ & --- & --- \\
\hline & 10 & $108 \pm 13^{\text {a }}$ & $77 \pm 6^{b}$ & $107 \pm 41^{\mathrm{a}}$ & $79 \pm 27^{\mathrm{a}}$ & --- & --- \\
\hline & 15 & $213 \pm 89^{a}$ & $156 \pm 65^{\mathrm{a}}$ & $54 \pm 21^{b}$ & $35 \pm 10^{b}$ & --- & --- \\
\hline & 20 & $209 \pm 66^{\mathrm{a}}$ & $81 \pm 29^{b}$ & $66 \pm 14^{b}$ & $41 \pm 3^{b}$ & --- & --- \\
\hline \multirow[t]{5}{*}{0.25} & 0 & $209 \pm 83^{\text {a }}$ & $199 \pm 80^{\mathrm{a}}$ & $82 \pm 21^{b}$ & $73 \pm 20^{b}$ & $26 \pm 7^{\mathrm{c}}$ & $18 \pm 6^{c}$ \\
\hline & 5 & $223 \pm 33^{a}$ & $211 \pm 31^{\mathrm{a}}$ & $91 \pm 4^{b}$ & $88 \pm 4^{b}$ & $48 \pm 4^{b}$ & $41 \pm 3^{\mathrm{b}}$ \\
\hline & 10 & $173 \pm 11^{\text {a }}$ & $163 \pm 10^{\mathrm{a}}$ & $93 \pm 14^{b}$ & $85 \pm 11^{b}$ & $53 \pm 4^{b}$ & $44 \pm 3^{b}$ \\
\hline & 15 & $233 \pm 14^{\mathrm{a}}$ & $217 \pm 11^{\mathrm{a}}$ & $100 \pm 40^{\mathrm{b}}$ & $91 \pm 38^{b}$ & $108 \pm 7^{\mathrm{a}}$ & $101 \pm 6^{\mathrm{a}}$ \\
\hline & 20 & $239 \pm 18^{\text {a }}$ & $229 \pm 17^{\mathrm{a}}$ & $207 \pm 3^{\mathrm{a}}$ & $201 \pm 2^{\mathrm{a}}$ & $125 \pm 16^{\mathrm{a}}$ & $113 \pm 14^{\mathrm{a}}$ \\
\hline \multirow[t]{5}{*}{0.5} & 0 & $261 \pm 51^{b}$ & $250 \pm 48^{b}$ & $136 \pm 8^{c}$ & $127 \pm 6^{\mathrm{c}}$ & $31 \pm 7^{c}$ & $20 \pm 7^{\mathrm{c}}$ \\
\hline & 5 & $324 \pm 54^{\mathrm{a}}$ & $309 \pm 52^{\mathrm{ab}}$ & $170 \pm 17^{b}$ & $164 \pm 15^{b}$ & $58 \pm 4^{b}$ & $50 \pm 1^{\mathrm{b}}$ \\
\hline & 10 & $230 \pm 49^{b}$ & $223 \pm 47^{b}$ & $195 \pm 23^{b}$ & $190 \pm 23^{b}$ & $47 \pm 6^{\text {bc }}$ & $41 \pm 5^{\mathrm{b}}$ \\
\hline & 15 & $268 \pm 28^{a b}$ & $259 \pm 25^{b}$ & $182 \pm 17^{b}$ & $173 \pm 10^{\mathrm{b}}$ & $102 \pm 2^{\mathrm{a}}$ & $96 \pm 1^{\mathrm{a}}$ \\
\hline & 20 & $359 \pm 17^{\text {a }}$ & $346 \pm 17^{\mathrm{a}}$ & $261 \pm 18^{a}$ & $246 \pm 11^{\mathrm{a}}$ & $100 \pm 9^{\mathrm{a}}$ & $90 \pm 6^{\mathrm{a}}$ \\
\hline \multirow[t]{5}{*}{1.0} & 0 & $221 \pm 29 \mathrm{bc}$ & $210 \pm 298^{b}$ & $175 \pm 9^{c}$ & $165 \pm 8^{c}$ & $36 \pm 5^{c}$ & $21 \pm 2^{d}$ \\
\hline & 5 & $373 \pm 16^{\mathrm{a}}$ & $357 \pm 14^{\mathrm{a}}$ & $147 \pm 24^{c}$ & $138 \pm 23^{c}$ & $38 \pm 2^{c}$ & $29 \pm 1^{\mathrm{cd}}$ \\
\hline & 10 & $415 \pm 6^{\mathrm{a}}$ & $403 \pm 7^{\mathrm{a}}$ & $199 \pm 21^{b}$ & $193 \pm 21^{\mathrm{c}}$ & $40 \pm 8^{c}$ & $30 \pm 4^{c}$ \\
\hline & 15 & $199 \pm 52^{c}$ & $192 \pm 49^{c}$ & $242 \pm 30^{\mathrm{ab}}$ & $236 \pm 29^{a}$ & $74 \pm 9^{\mathrm{a}}$ & $62 \pm 6^{\mathrm{a}}$ \\
\hline & 20 & $274 \pm 32^{b}$ & $263 \pm 32^{b}$ & $273 \pm 27^{\mathrm{a}}$ & $264 \pm 24^{b}$ & $62 \pm 10^{b}$ & $43 \pm 4^{b}$ \\
\hline
\end{tabular}

$\mu \mathrm{M}$. With increasing S supply to plants, an obvious reduction in Se uptake was found. However, Se uptake in plants supplied with $0.5 \mathrm{mM}$ (cabbage) or $1.0 \mathrm{mM}$ (kohlrabi) $\mathrm{S}$, was rather increased in comparison with lower $\mathrm{S}$ levels. In contrast to cabbage and kohlrabi, a continuous reduction of Se uptake was observed with increasing supply of $\mathrm{S}$ in alfalfa (Fig. 1).

A great proportion i.e. up to $97 \%$ of Se taken up, was transported into shoot. This proportion did not significantly change in response to increasing $\mathrm{S}$ supply. In alfalfa plants at the highest Se concentration $(20 \mu \mathrm{M})$, transport of Se reached up to 40 $\mu \mathrm{g} \mathrm{g}^{-1}$ root DW, which was higher than the highest values in two varieties of Brassica (Fig. 2).

As expected, Se volatilization increased with increasing Se concentration in the medium. A distinctive metallic odor characteristic of Se was detected in the growth chamber during the experiment. The amount of volatilized Se based on leaf area was much higher in alfalfa than two varieties of Brassica. When was expressed per plant, however, three studied varieties/species were similar in Se volatilization (Fig. 3).

\section{Discussion}

\section{Dual effect of Se on growth}

Depending on S nutritional status of plants, Se exerted either an inhibitory or a stimulatory effect on growth. However, the extent of both toxic and beneficial effect of Se on growth parameters was 
Vol. 17 (2008): 177-190.
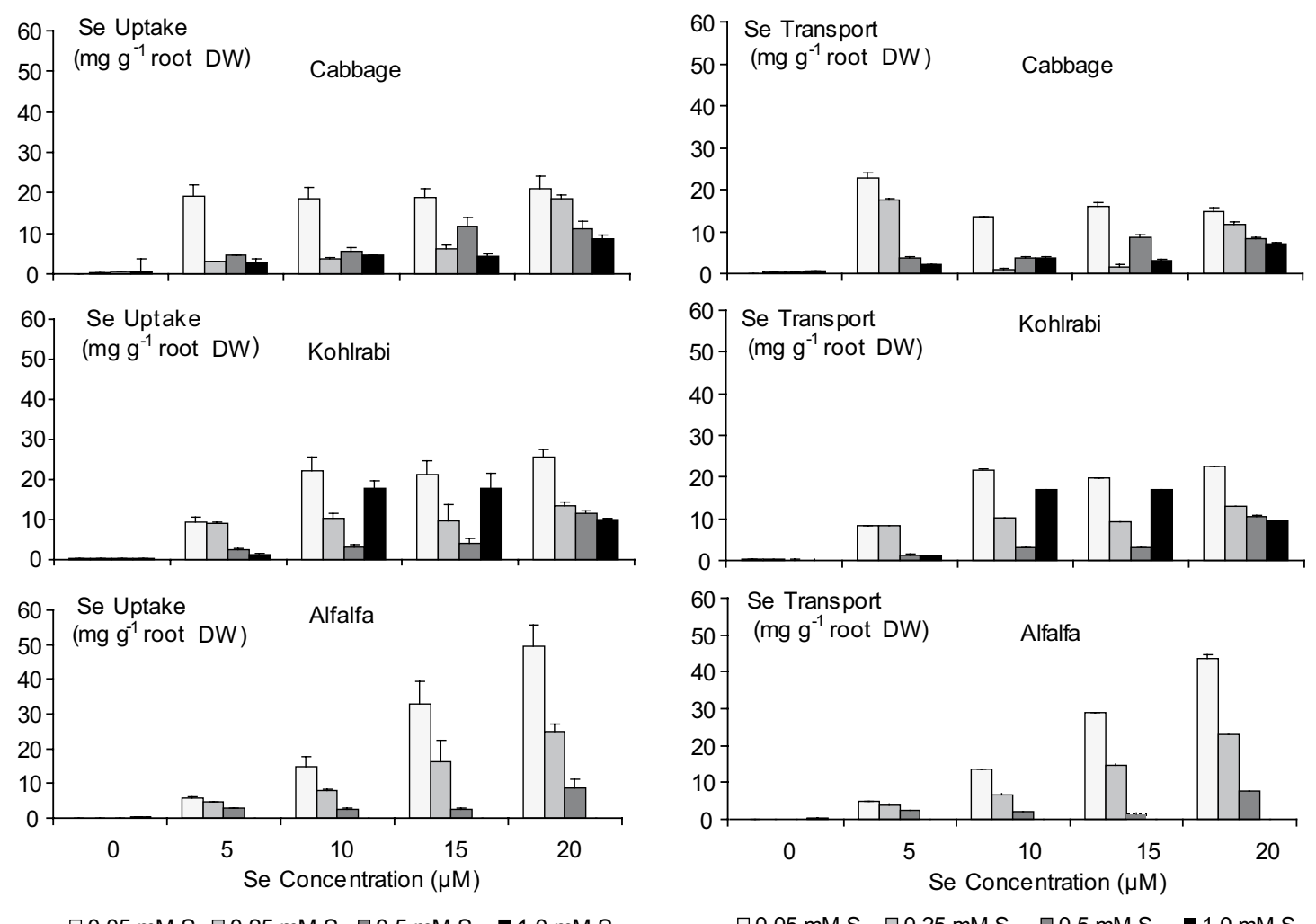

Fig 1. Changes in the Se uptake ( $\mathrm{mg} \mathrm{g}^{-1}$ Root DW) at different nutritional status of S in cabbage (Brassica oleracea L. var. Sabauda), kohlrabi (Brassica oleracea L. var. Gongyloides) and alfalfa (Medicago sativa L. cv. Garehyounjeh) treated by different levels of Se. Error bars indicate SD.

Fig 2. Changes in the Se transport ( $\mathrm{mg} \mathrm{g}^{-1}$ Root DW) at different nutritional status of S in cabbage (Brassica oleracea L. var. Sabauda), kohlrabi (Brassica oleracea L. var. Gongyloides) and alfalfa (Medicago sativa L. cv. Garehyounjeh) treated by different levels of Se. Error bars indicate SD.

\section{$\square 0 \mu \mathrm{M}$ Se $\quad \square 10 \mu \mathrm{M}$ Se $\square 20 \mu \mathrm{M} \mathrm{Se}$}

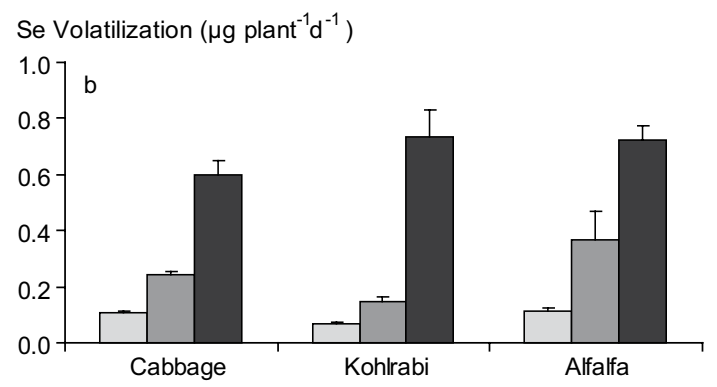

Plant Varieties/Species

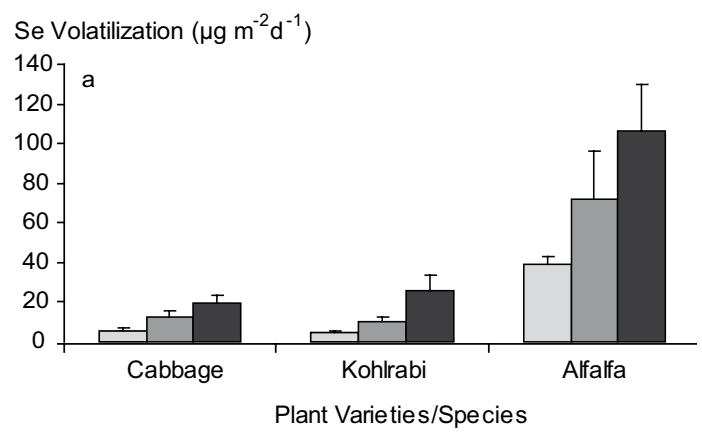

Fig 3. Se volatilization in cabbage (Brassica oleracea L. var. Sabauda), kohlrabi (Brassica oleracea L. var. Gongyloides) and alfalfa (Medicago sativa L. cv. Garehyounjeh) treated by different levels of $\mathrm{Se}(\mathrm{S}=1.0 \mathrm{mM})$ on the basis of leaf area $\left(\mu \mathrm{g} \mathrm{m}^{-2} \mathrm{~d}^{-1}\right)$ and per plant $\left(\mu \mathrm{g}\right.$ plant $\left.{ }^{-1} \mathrm{~d}^{-1}\right)$. Error bars indicate SD. 
Hajiboland, R. \& Amjad, L. Accumulation and volatilization of selenium in two Brassica varieties

highly dependent on plant species/varieties. For example a significant growth stimulatory effect of 5 $\mu \mathrm{M}$ Se was observed in strongly S deficient kohlrabi $(0.05 \mathrm{mM})$ while alfalfa plants died at the same treatments. The toxic effect of Se to plants results mainly from interferences of Se with $\mathrm{S}$ metabolism and replacing S-amino acids by corresponding Seamino acids and their subsequent incorporation into proteins (Terry et al. 2000). Different tolerance to Se toxicity of Brassica varieties compared with alfalfa in this work is likely the result of differences in the extent of Se-amino acids formation and incorporation into proteins and/or different production of nonproteinogenic methylated Seamino acids. In addition of Se hyperaccumulators, in some secondary accumulators such as broccoli (Brassica oleracea var. Italica) (Lyi et al. 2005) the accumulation of these non toxic species of Se-amino acids has been suggested to be the basis of Se tolerance (Neuhierl et al. 1999). It could be suggested that studied varieties of $B$. oleracea in this work possess similar mechanism for prevention of Se toxicity. Further studies are necessary for proving of involving biochemical pathways in these varieties.

Among growth parameters, root length and leaf area with up to $82 \%$ and $83 \%$ reduction in cabbage and $74 \%$ and $58 \%$ reduction in kohlrabi respectively, were the most sensitive parameters to Se toxicity. A high susceptibility of root length and leaf area could be likely explained by a direct effect of Se toxicity on cell division and/or expansion of cells.

Enhancement of growth at low Se concentrations demonstrated in this work was also observed in Arabidopsis (White et al., 2004) and rye grass (Hartikainen et al. 2000). However, the growth improvement in this work was much higher than that reported for other species e.g. $8 \%$ increase for shoot DW in ryegrass (Hartikainen et al. 2000). Growth improvement due to Se supplementation, was much higher in cabbage than kohlrabi and alfalfa. The positive growth response of plants to Se added at small concentrations, have been attributed to the antioxidative effect of Se (Hartikainen et al. 2000).

\section{Se and $S$ uptake and transport}

Supply of plants with increasing concentrations of S, reduced Se uptake and transport. Because of chemical similarities, sulphate $\left(\mathrm{SO}_{4}^{-2}\right)$ and selenate $\left(\mathrm{SeO}_{4}^{-2}\right)$ ions may be in direct competition for the same binding sites in plants during active absorption (White et al. 2004). Another cause of inhibition of selenate uptake by high external sulphate is related to the reduction of selenate activity by sulphate in the external solution as was calculated by Shennan et al. (1990).

In contrast to the inhibitory effect of $\mathrm{S}$ on $\mathrm{Se}$ uptake, rather a significant enhancement of $S$ uptake and proportion of transported $\mathrm{S}$ into shoot was observed in Se treated plants. Similar effect of Se on $\mathrm{S}$ uptake and accumulation was observed by other authors (Kopsell and Randle 1999, White et al. 2004). It seems likely that an antagonistic effect of Se on S uptake and transport have been reported only in experiments with high $\mathrm{SeO}_{4}{ }^{2-}: \mathrm{SO}_{4}{ }^{2-}$ ratio in the medium e.g. a $1 \mathrm{SeO}_{4}{ }^{2-}: 1 \mathrm{SO}_{4}{ }^{2-}$ ratio by Ferrari and Renosto (1972). In experiments used lower ratio of Se to S e.g. 1:4 and 1:2 in the nutrient solution, $\mathrm{S}$ concentration in plants increased with increasing Se concentrations (Kopsell and Randle 1999). The cause of stimulatory effect of selenate on S uptake and transport is not known exactly, but it was suggested that rhizosphere selenate promotes sulphate uptake, possibly by preventing the reduction in the abundance and/or activity of sulphate transporters by sulphate and/or its metabolism (White et al. 2004).

In severely $\mathrm{S}$ deficient cabbage and kohlrabi (at $0.05 \mathrm{mM} \mathrm{S}$, remind at this level of S, alfalfa plants were died in the presence of Se as low as $5 \mu \mathrm{M}$ ), Se uptake and transport was very high and did not respond to increasing Se concentration in the medium. A response to Se concentration was observed only in plants supplied with $0.25 \mathrm{mM} \mathrm{S}$ and higher. It could be attributed to a passive Se influx from medium to root and then transport into shoot. S is one of the important structural components of plasma membranes (Marschner 1995) and concentration of sulfolipids was reported to reduce by about two folds in S-starved plants (Nikiforova et al 2005). Therefore, it could be suggested that, sever S deficiency results in loss of membrane integrity and therefore 
Vol. 17 (2008): 177-190.

passive fluxes of ions. It is well known that, production of reactive oxygen species and reduction of antioxidant enzymes damages membrane integrity (Elstner 1982). Results of our previous experiment demonstrated that sever S deficiency inhibit activity of ascorbat peroxidae, catalase and superoxide dismutase and increased $\mathrm{H}_{2} \mathrm{O}_{2}$ concentration (Hajiboland and Amjad 2007). There are numerous reports on the induction of oxidative stress and loss of membrane integrity due to nutrients deficiency ( $\mathrm{Yu}$ et al. 1998, Candan and Tarhan 2003).

An unusual increase in the uptake and transport of Se was observed in cabbage (supplied with $0.5 \mathrm{mM} \mathrm{S}$ ) and kohlrabi (supplied with1.0 mM S) compared with plants treated with rather higher Se and lower S concentrations. These changes are likely the result of the effect of $\mathrm{S}$ nutritional status on affinity of S carriers for Se. White et al (2004) suggested that Se and S enter Arabidopsis through multiple transport pathways with contrasting $\mathrm{S} / \mathrm{Se}$ selectivity, whose activities vary between plants of contrasting nutritional status. For examination of this hypothesis for studied species/varieties in this work a short-term uptake experiment is necessary.

The translocation of high proportion of $\mathrm{Se}$ taken up from root to shoot was observed in this work indicates a low potential of roots for incorporation of selenate into organic molecules. It was shown that in contrast to selenite, plants transport and accumulate substantial amounts of selenate in leaves (Zayed et al. 1998) likely because it is not readily converted to organic forms of Se in roots (Terry et al. 2000).

\section{Se accumulation and volatilization}

Se accumulated to a great extent in S deficient plants, which was the result of both increased uptake and transport of Se and concentration effect due to $\mathrm{S}$ deficiency- induced growth inhibition. S deficient cabbage, kohlrabi and alfalfa plants accumulated up to $1.2,9.1$ and $4.3 \mathrm{mg} \mathrm{g}^{-1} \mathrm{DW}$ Se in shoot and 6.6, 1.4 and $5.8 \mathrm{mg} \mathrm{g}^{-1} \mathrm{DW}$ Se in root. The maximum values of Se concentration of S sufficient plants were
0.4, 1.6 and $2.2 \mathrm{mg} \mathrm{g}^{-1} \mathrm{DW}$ for shoot and 1.4, 0.9 and $2.0 \mathrm{mg} \mathrm{g}^{-1} \mathrm{DW}$ for root of cabbage, kohlrabi and alfalfa respectively. Se accumulation in dry matter of leaves in various Brassica varieties treated by 20 $\mu \mathrm{M}$ selenate in the nutrient solution, was reported to be $0.15-0.5 \mathrm{mg} \mathrm{g}^{-1} \mathrm{DW}$ (Souza et al. 2002) which are in the range or slightly lower than observed amounts in S sufficient plants in this work.

The average Se concentration of crop plants from non-seleniferous soils varies between about 0.01 and $1.0 \mathrm{mg} \mathrm{kg}^{-1} \mathrm{DW}$ (Marschner 1995) e.g. approximately 1000 times lower than the Se amounts in this work. The Se concentration of shoot in soil grown ryegrass treated by $13 \mu \mathrm{mol} \mathrm{kg}{ }^{-1}$ soil $(1 \mathrm{mg}$ $\mathrm{kg}^{-1}$ ) was reported to be $16 \mu \mathrm{g} \mathrm{g}^{-1} \mathrm{DW}$ (Hartikainen et al. 2000) which was much lower than values observed at similar treatments in this work, e.g. 211-1565 $\mu \mathrm{g} \mathrm{g}^{-1}$ DW. Using soils contaminated with moderate levels of Se, it was shown that some Brassica species (B. juncea and B. napus) could be classified as secondary Se accumulator plants with a typical Se concentration of several hundred $\mathrm{mg}$ of Se kg-1 DW in their shoot tissues (Bañuelos et al. 1997).

For hydroponically grown plants, critical toxic Se concentration in the shoot was determined at 320-330 $\mu \mathrm{g} \mathrm{g}^{-1}$ DW for ryegrass and white clover (Smith and Watkinson 1984). Other authors reported significantly higher amounts, e.g. up to 840-900 $\mu \mathrm{g} \mathrm{g}^{-1}$ DW for ryegrass and strawberry clover (Hopper and Parker, 1999). Studied plants in this work showed rather a growth promotion at tissue concentrations as high as 446 (cabbage), 771 (kohlrabi) and 883 (alfalfa) $\mu \mathrm{g} \mathrm{g}^{-1} \mathrm{DW}$.

Unexpectedly, Se volatilization based on dry matter of leaf (not shown) and leaf area was higher in alfalfa than two varieties of Brassica. It means that, the activity of enzymes involving in the formation of volatilized Se is higher in alfalfa than cabbage and kohlrabi. However, because of different biomass, the amounts of volatilized Se expressed as $\mu \mathrm{g}$ per plant, were similar among three studied species/varieties. The volatilized Se in some varieties of Brassica was reported to be 0.2-0.8 $\mu \mathrm{g}$ $\mathrm{g}^{-1} \mathrm{DW}$ (Souza et al. 2002) which is in the range of our observed data for cabbage and kohlrabi but much lower than amounts determined for alfalfa. 
Hajiboland, R. \& Amjad, L. Accumulation and volatilization of selenium in two Brassica varieties

Phytovolatilization is an important remediation process because it prevents Se from entering the food chain. Se is volatilized mostly as dimethylselenide (Wilber 1980).

Se has long been recognized to be an essential micronutrient for animals and human. To

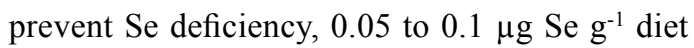
is required, Se toxicosis in animals is the consequence when dietary concentrations of Se exceed 5 to $15 \mu \mathrm{g} \mathrm{g}^{-1}$ (Mayland et al. 1989). These critical concentrations were exceeded by all treatments in this experiment and are consisted with the ability of these plants to accumulate Se, therefore, these plants cannot be used without dilution.

The vegetable Brassica have been identified as good delivery system for organic Se in human diets (Kopsell and Randle 1999). Results of this work show that, alfalfa could be considered as one suitable plant species for Se supplementation of livestock fodder. However, adequate $\mathrm{S}$ nutrition of plants is necessary in order to reduce the risk of Se toxicity for animals and human.

\section{Conclusion}

Lower susceptibility to toxic concentrations of Se in combination with greater growth stimulation by supplemental Se in two Brassica varieties compared with alfalfa reveals a mechanism functioning only in Brassicaceae. Such mechanism may facilitate an efficient internal detoxification of Se as well as higher efficiency for utilization of this element in growth improvement. Due to the presence of glucosinolates in family Brassicacea, they could be the candidate buffering molecules. These molecules likely trap excess Se particularly in the absence of enough $\mathrm{S}$ in plant tissues and exclude Se from protein fractions. Prevention of toxicity may allow plants to recruit low levels of free Se for growth likely via activation of antioxidant defence system, enhancement of reduced cell metabolites or change of lignification and auxin metabolism.

\section{References}

Bañuelos, G.S., Ajwa, H.A., Wu, L., Guo, X., Akohoue, S. \& Zambrzuski, S. 1997. Selenium-induced growth reduction in Brassica land races considered for phytoremediation. Ecotoxicology and Environmental Safety 36:282-287.

Broadley, M.R., White, P.J., Bryson, R.J., Meacham, M.C., Bowen, H.C., Johnson, S.E., Hawkesford, M.J., McGrath, S.P., Zhao, F-J, Breward, N., Harriman, M. \& Tucker, M, 2006. Biofortification of UK food crops with selenium. Proceedings of the Nutrition Society 65:169-181.

Ebbs, S. \& Weinstein, L. 2001. Alteration of selenium transport and volatilization in barley (Hordeum vulgare) by arsenic. Journal of Plant Physiology 158:1231-1233.

Ellis, D.R. \& Salt, D.E. 2003. Plants, selenium and human health. Current Opinion in Plant Biology 6:273-279.

Candan, N. \& Tarhan, L. 2003. The correlation between antioxidant enzyme activities and lipid peroxidation levels in Mentha pulegium organs grown in $\mathrm{Ca}^{2+}, \mathrm{Mg}^{2+}$, $\mathrm{Cu}^{2+}, \mathrm{Zn}^{2+}$ and $\mathrm{Mn}^{2+}$ stress conditions. Plant Science 165:769-776.

Combs, G.F. 1989. Selenium. In: Moon, T.E. \& Micozzi, M.S. (eds.). Nutrition and cancer prevention: Investigation the role of micronutrients. Marcel Dekker: New York, USA. p. 389-419.

Ekholm, P. 1997. Effects of selenium supplemented commercial fertilizers on food selenium contents and selenium intake in Finland. EKT-Series No. 1047. Helsinki, Academic dissertation, University of Helsinki.

Elstner, E.F. 1982. Oxygen activation and oxygen toxicity. Annual Review of Plant Physiology 33:73-96.

Ferrari, G. \& Renosto, F. 1972. Regulation of sulphate uptake by excised barley roots in the presence of selenate. Plant Physiology 49:114-116.

Gissel-Nielsen, G., Gupta, U.C., Lamand, M. \& Westermarck, T. 1984. Selenium in soils and plants and its importance in livestock and human nutrition. Advances in Agronomy 37:397-461.

Grieve, C.M., Suarez, D.L. \& Shannon, M.C. 1999. Effect of saline irrigation water composition on selenium accumulation by wheat. Journal of Plant Nutrition 22:1443-1450.

Gupta, P.K. 1999. Soil, Plant, Water and Fertilizer Analysis. Agrobios: Jodhpur, India.

Hajiboland, R. \& Amjad, L. 2007. Does antioxidant capacity of leaves play a role in growth response to selenium at different sulphur nutritional status? Plant, Soil and Environment 53:207-215.

Hartikainen, H, Xue, T. \& Piironen, V. 2000. Selenium as an anti-oxidant and pro-oxidant in ryegrass. Plant and Soil 225:193-200.

Hoagland, D.R. \& Arnon, D.I. 1950. The water culture method for growing plants without soil. California Agricultural Experiments Station Circular 347. Berkeley, CA.

Hobbins, W.B. 1981. Selenium determination by hydride generation. Varian Instruments at Work AA-11. Varian Instruments Pty. Ltd., Mulgrave, Victoria, Australia.

Hopper, J.L. \& Parker, D.R. 1999. Plant availability of selenite and selenate as influenced by the competing ions phosphate and sulphate. Plant and Soil 210:199-207. 


\section{AGRICULTURAL AND FOOD SCIENCE}

Vol. 17 (2008): 177-190.

Kopsell, D. \& Randle, W.M. 1999. Selenium accumulation in a rapid-cycling Brassica oleracea population responds to increasing sodium selenate concentrations. Journal of Plant Nutrition 22:927-937.

Lewis, B.G., Johnson, C.M. \& Delwiche, C.C. 1966. Release of volatile selenium compounds by plants: collection procedures and preliminary observations. Journal of Agricultural and Food Chemistry 14:638-640.

Lyi, S.M., Heller, L., Rutzke, M., Welch, R.M., Kochian, L.V. \& Li, L. 2005. Molecular and biochemical characterization of the selenocysteine Se-methyltransferase gene and Se-methylselenocysteine synthesis in broccoli. Plant Physiology 138: 409-420.

Marschner, H. 1995. Mineral Nutrition of Higher Plants. $2^{\text {nd }}$ edition. Academic press: London.

Mayland, H.F., James, L.F., Panter, K.E. and Sonderegger, J.L. 1989. Selenium in seleniferous environments. In: Jacobs, L.W. (ed.). Selenium in Agriculture and the Environment. American Society of Agronomy: Madison, Wisconsin. p. 15-50.

Mikkelsen, R.L, Haghnia G.H. \& Page AL 1987. Effects of $\mathrm{pH}$ and selenium oxidation state on the selenium accumulation and yield of alfalfa. Journal of Plant Nutrition 10:937-950.

Mikkelsen, R.L., Page, A.L., Haghnia, G.H. 1988. Effect of salinity and its composition on the accumulation of selenium by alfalfa. Plant and Soil 107:63-67.

Neuhierl, B., Thanbichler, M., Lottspeich, F. \& Bock, A. 1999. A family of S-methylmethionine-dependent thiol/ selenol methyltransferases. Role in selenium tolerance and evolutionary relation. Journal of Biological Chemistry 274:5407-5414.

Nikiforova, V.J., Kopka, J., Tolstikov, V., Fiehn, O., Hopkins, L., Hawkesford, M.J., Hesse, H. \& Hoefgen R. 2005. Systems rebalancing of metabolism in response to sulfur deprivation, as revealed by metabolome analysis of Arabidopsis Plants. Plant Physiology 138:304-318.

Norheim, G. \& Haugen, A. 1986. Precise determination of selenium in tissues using automated wet digestion and an automated hydride generator-atomic absorption spectrometry. Fresenius Zeitschrift für Analytische Chemie 279:101-105.

Shennan, C., Schachtman, D.P. \& Cramer, G.R. 1990. Variation in $\left[{ }^{75} \mathrm{Se}\right]$ selenate uptake and partitioning among tomato cultivars and wild species. New Phytologists 115: 523-530.

Shrift, A. 1969. Aspects of selenium metabolism in higher plants. Annual Review of Plant Physiology 20: 475-494.

Smith, G.S. \& Watkinson, J.H. 1984. Selenium toxicity in perennial ryegrass and white clover. New Phytologists 97:557-564.

Soltanpour, P.N. \& Workman S.M. 1980. Use of $\mathrm{NH}_{4} \mathrm{HCO}_{3}-$ DTPA soil test to assess availability and toxicity of selenium to alfalfa plants. Communications in Soil Science and Plant Analysis 11:1147-1156.

Sors, T.G., Ellis, D.R. \& Salt, D.E. 2005. Selenium uptake, translocation, assimilation and metabolic fate in plants. Photosynthesis Research 86:373-389.

Souza, M.P., Pickering, I.J., Walla, M. \& Terry, N. 2002. Selenium assimilation and volatilization from selenocyanate-treated Indian mustard and muskgrass. Plant Physiology 128:625-633.

Suarez, D.L., Grieve, C.M. \& Poss, J.A. 2003. Irrigation method affects selenium accumulation in forage Brassica species. Journal of Plant Nutrition 26:191-201.

Tennant, D. 1975. A test of modified line intersect method of estimating root length. Journal of Ecology 63:995-1001.

Terry, N., Zayed, A.M., Souza, M.P. \& Tarun, A.S. 2000. Selenium in higher plants. Annual Review of Plant Physiology and Plant Molecular Biology 51:401-432.

White, P.J., Bowen, H.C., Parmaguru, P., Fritz, M., Spracklen, W.P., Spiby, R.E., Meacham, M.C., Mead, A., Harriman, M., Trueman, L.J., Smith, B.M., Thomas, B. \& Broadley, M.R. 2004. Interactions between selenium and sulfur nutrition in Arabidopsis thaliana. Journal of Experimental Botany 55:1927-1937.

Wilber, C.G. 1980. Toxicology of selenium: a review. Clinical Toxicology 17:171-230.

Yu, Q., L. Osborne \& Rengel, Z. 1998. Micronutrient deficiency changes activities of superoxide dismutase and ascorbate peroxidase in tobacco plants. Journal of Plant Nutrition 21:1427-1437.

Zayed, A.M., Lytle, C.M. \& Terry, N. 1998. Accumulation and volatilization of different chemical species of selenium by plants. Planta 206:284-292. 
